\title{
Series de clima en anillos de Aspidosperma polyneuron Müll. Arg. y Anacardium excelsum (Bertero ex Kunth) Skeels
}

\author{
Climate series in rings of Aspidosperma polyneuron Müll.Arg. y \\ Anacardium excelsum (Bertero ex Kunth) Skeels
}

\author{
Ana María Briceño-j ${ }^{1 *}{ }^{\circledR}$ y Jesús Orlando Rangel-Ch ${ }^{1}$
}

Briceño-J. A.M. y Rangel-Ch, J.O. (2021). Series de clima en anillos de Aspidosperma polyneuron Müll.Arg. y Anacardium excelsum (Bertero ex Kunth) Skeels. Colombia Forestal, 24(2), 52-64.

Recepción: 7 de mayo 2020

\section{Resumen}

Se analizó la dinámica de crecimiento de Aspidosperma polyneuron y Anacardium excelsum, especies que crecen en el bosque seco tropical en el departamento del Cesar. Se utilizaron técnicas dendrocronológicas y modelos de regresión (Clench, Exponencial negativa, Logístico y Gompertz). Los resultados obtenidos muestran que los anillos de $A$. polyneuron permitieron construir una cronología de 161 años (1854-2014) y los de $A$. excelsum de 128 años (1887-2014). El crecimiento de $A$. polyneuron mostró una respuesta positiva con la precipitación espontánea en los meses secos, mientras que A. excelsum la mostró en los meses más lluviosos. La temperatura y el SOI (Índice de Oscilación Sur) no mostraron correlaciones con el crecimiento. Los anillos de A. polyneuron y $A$. excelsum documentaron variaciones climáticas locales pero no globales, como el SOI. De acuerdo a los modelos de regresión las dos especies tardarán más de 100 años en alcanzar el diámetro máximo, por lo que es preciso pensar estrategias para la conservación de las especies y la deforestación evitada.

Palabras clave: anillos de crecimiento, bosque seco tropical, caribe colombiano, precipitación, temperatura, SOI.
Aprobación: 9 de abril 2021

\begin{abstract}
The growth dynamics of Aspidosperma polyneuron and Anacardium excelsum species that grow in the tropical dry forest of the Cesar department were analyzied. We used dendrochronological techniques and regression models (Clench, Negative Exponential, Logistic and Gompertz). The results obtained show that $A$. polyneuron rings allowed to build a chronology of 161 years (1854-2014) and the rings of $A$. excelsum a chronology of 128 years (1887-2014). The growth of $A$. polyneuron showed a positive growth with spontaneous rain in the dry months and $A$. excelsum with the rainiest months. Temperature and SOI did not show correlations with growth. The rings of $A$. polyneuron and A. excelsum documented local climatic variations, but not global as SOI. According to the regression models, the two species will take more than 100 years to reach the maximum diameter, so it is necessary to think about the conservation of the species and the avoided deforestation.
\end{abstract}

Keywords: growth rings, tropical dry forest, Colombian Caribbean, precipitation, temperature, SOI.

1 Instituto de Ciencias Naturales, Universidad Nacional de Colombia, Bogotá, Colombia.

ambricenoj@unal.edu.co. Autora para correspondencia. 


\section{INTRODUCCIÓN}

El monto anual de la lluvia y su régimen de distribución influyen en la composición florística y en la estructura y arquitectura de la vegetación, como se ha mencionado en la caracterización de la vegetación de varias regiones naturales de Colombia (Minorta-C. et al., 2019; Rangel-Ch., 2012). Bajo un escenario de cambio climático, un desmejoramiento en los patrones de lluvia (montos y régimen de distribución) tendría efectos drásticos sobre la vegetación natural y los cultivos agrícolas, especialmente en las regiones secas. Las variaciones en la temperatura superficial del mar en el océano Pacífico, por ejemplo, se manifiestan en anomalías que afectan la dinámica del clima continental, especialmente en la franja tropical (Fedorov y Philander, 2000), donde se producen periodos muy lluviosos con descensos de la temperatura o periodos con escasas Iluvias y aumentos de la temperatura. Estos fenómenos se denominan La Niña y El Niño y se les ha asociado al Índice de Oscilación del Sur (SOI) (Bendix et al., 2011).

Con base en la información disponible para el último siglo, desde 1970 se ha registrado una mayor cantidad de años en los cuales las condiciones del fenómeno del Niño están presentes (Berliner, 2003; Malhi y Wright, 2004). En Colombia la manifestación de este fenómeno provoca la disminución de la precipitación con menor disponibilidad de agua y periodos intensos de sequía (Rangel-Ch. y Carvajal-Cogollo, 2009).

Las relaciones entre las variaciones de los parámetros climáticos, como la precipitación y la temperatura, influyen en el crecimiento de las especies arbóreas. Dicha relación puede ser documentada mediante técnicas dendrocronológicas (Hughes, 2011) que tienen como uno de sus objetivos principales la reconstrucción del clima pasado. Una condición apropiada para realizar estos estudios y analizar las variaciones del clima es la formación de anillos de crecimiento anuales (Oliveira et al., 2009; Pereyra et al., 2014). Si tomamos el caso de las regiones tropicales, vemos que ha aumentado la lista de especies de las que se ha documentado la manifestación de crecimiento anual de los anillos (Groenendijk et al., 2014; Tomazello-Filho et al., 2000, 2009) con importantes implicaciones para la ecología y el manejo forestal (Stahle et al., 1999). Estas estimaciones han considerado la realización de modelos matemáticos de crecimiento de las especies como von Bertalanffy, y en especial de especies con valor comercial por su implicación en el sector maderero (Del Valle, 1997). Respecto al manejo forestal del crecimiento de las especies, este ha sido descrito mediante modelos matemáticos en los que la mayoría de los estudios sobre los modelos en el trópico se han concentrado en especies foráneas y nativas cuya madera tiene valor comercial (Del Valle, 1997), como es el caso de: Gmelina arborea Roxb., Acrocarpus fraxinifolius Arn., Tectona grandis L.f. (Muñoz et al., 2009), Ochroma pyramidale (Cav. ex Lam.) Urb., Ochroma lagopus Sw; (Suatunce et al., 2009), Terminalia amazonia (J.F.Gmel.) Exell, Hieronyma alchorneoides Allemăo, Vochysia ferruginea Mart. y Vochysia guatemalensis Donn. Sm. (Arias, 2005).

En la región del Caribe colombiano, las áreas con bosque seco tropical se caracterizan porque la evapotranspiración potencial supera a la precipitación durante la mayor parte del año (Rangel-Ch. y Carvajal-Cogollo, 2009). Estos bosques, afectados por el déficit hídrico, son muy sensibles a los cambios en los montos y en los regímenes de distribución en las lluvias, y sus condiciones desfavorables se agudizan cuando se presentan sequías severas (Rangel-Ch. y Carvajal-Cogollo, 2009). Entre las especies arbóreas de importancia por su dominancia en la vegetación de la zona de estudio figura Aspidosperma polyneuron Muell. Arg., de amplia distribución en Suramérica. En Colombia tiene presencia en el Caribe, alrededor de los complejos cenagosos de Zapatosa y el Sur del Cesar, y en la parte baja de la Serranía de Perijá, extendiéndose en algunos casos al sector norte del valle del río Magdalena. Dado que esta especie es explotada en exceso por el sector maderero para la construcción de vivienda y en ebanistería 
para la construcción de muebles y pisos, las poblaciones actuales están seriamente amenazadas debido a la reducción drástica de su hábitat original. Es por esto que, dentro del plan de conservación y manejo de la especie, la Corporación Autónoma Regional de Cundinamarca (CAR) la ha categorizado en peligro, pues el $60 \%$ de su localidad en Colombia ha tenido una explotación intensa (Rodríguez, 2015). Por otra parte, Anacardium excelsum se distribuye desde Costa Rica hasta Brasil y en Colombia desde áreas húmedas en las serranías del Chocó biogeográfico hasta zonas semihúmedas en sectores del Parque Nacional Tayrona y los Montes de María (Córdoba). En la región andina, además, forma bosques homogéneos que se establecen en las orillas de quebradas y ríos. Sin embargo, debido a que su madera tiene una alta demanda para la elaboración de canoas, muebles sencillos, cajas livianas, construcción de viviendas rurales, carpintería, ebanistería y pisos, su explotación ha diezmado extensos territorios, como es el caso en las poblaciones de la Serranía del Darién y en la región tropical andina (Rangel-Ch., 2012).

Por lo anterior, esta investigación tuvo como objetivo: i) analizar el crecimiento radial y su relación con las variables climáticas (temperatura y precipitación) y el impulsador climático del fenómeno El Niño Oscilación del Sur (ENSO), documentado en el SOI, y ii) proponer un modelo de crecimiento radial para Aspidosperma polyneuron y Anacardium excelsum en el bosque seco tropical.

\section{MATERIALES Y MÉTODOS}

\section{Zona de estudio}

La fase de campo se realizó en la hacienda Río de Janeiro (Valledupar, Norte del departamento del Cesar, $10^{\circ} 31^{\prime} 04.3^{\prime \prime} \mathrm{N}, 73^{\circ} 10^{\prime} 38.6^{\prime \prime} \mathrm{W}$ ) y en el sector de la Bocatoma (Aguachica, Sur del Cesar, $\left.08^{\circ} 21^{\prime} 11.5^{\prime \prime} \mathrm{N}, 73^{\circ} 34^{\prime} 46.4^{\prime \prime}\right)$. En la zona de estudio los suelos son moderadamente profundos y ácidos, en la textura predominan las condiciones: arcillosa, franco-limosa y arenosa-franca, y el $\mathrm{pH}$ fluctúa entre 4.1 (muy ácido) y 9.9 (alcalino); además, la capacidad de intercambio catiónico está entre muy baja a baja y son muy bajos los contenidos de Carbono, Magnesio y Potasio (Rangel-Ch. y Carvajal-Cogollo, 2012a).

El tipo de clima de Valledupar (estación: Aeropuerto Alfonso López, $10^{\circ} 26^{\prime} \mathrm{N}, 73^{\circ} 15^{\prime} \mathrm{W}$, $138 \mathrm{~m}$ de altitud.), según la clasificación de Thornthwaite, es $\mathrm{DS}_{2} \mathrm{~A}^{\prime}$, semiárido con marcada deficiencia de agua en la época más seca (diciembre a marzo), y megatermal, con un valor máximo de evapotranspiración potencial (ETP) en marzo (Rangel-Ch. y Carvajal-Cogollo, 2012b). El monto anual de las precipitaciones en la serie de 1970 a 2013 fue de $997.2 \mathrm{~mm}$ con promedio mensual de $82.8 \mathrm{~mm}$ y una temperatura media de $29^{\circ} \mathrm{C}$ (Figura 1a). El régimen de precipitación es bimodal-tetraestacional. Los periodos Iluviosos de mayor intensidad van de agosto a noviembre y de mayo a junio; los meses de mayor pluviosidad son mayo $(169 \mathrm{~mm})$ y octubre $(197 \mathrm{~mm})$. El periodo seco de más intensidad va de diciembre a abril, con el valor más bajo en febrero (Figura 1b).

Para el caso de Aguachica (estación: Aguas Claras, $8^{\circ} 15^{\prime} \mathrm{N}, 73^{\circ} 37^{\prime} \mathrm{W}, 208 \mathrm{~m}$ de altitud), según la clasificación de Thornthwaite, el tipo de clima es $\mathrm{DS}_{2} \mathrm{~A}^{\prime}$, semiárido con deficiencia de agua en la época más seca (diciembre a marzo). El monto anual de precipitación para el periodo de 1974 a 2013 fue de $1364.3 \mathrm{~mm}$ con promedio mensual de $113.8 \mathrm{~mm}$ y una temperatura promedio de $28.3{ }^{\circ} \mathrm{C}$ (Figura 1c). El régimen de precipitación es unimodal-biestacional. El periodo Iluvioso va de abril a octubre; septiembre es el mes más Iluvioso (Rangel-Ch. y Carvajal-Cogollo, 2012b). El periodo seco va de noviembre a marzo, con el valor más bajo en enero (Figura $1 \mathrm{~d}$ ).

Muestreo. Tanto de A. polyneuron -en la reserva Río de Janeiro- como de $A$. excelsum -en la Bocatoma- se colectaron 45 rodajas basales: este número fue considerado porque superaba el valor 
a.

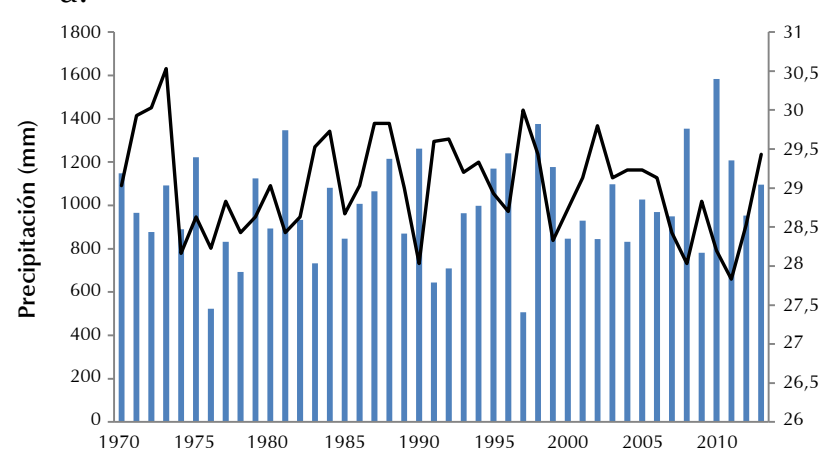

C.

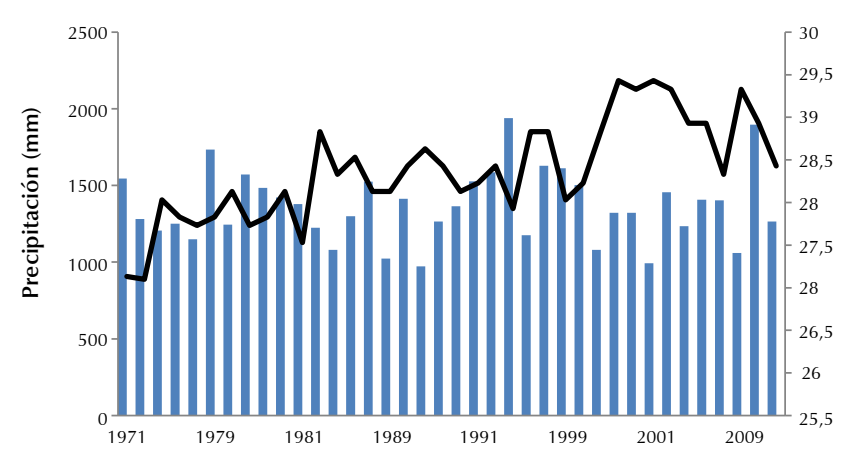

b.

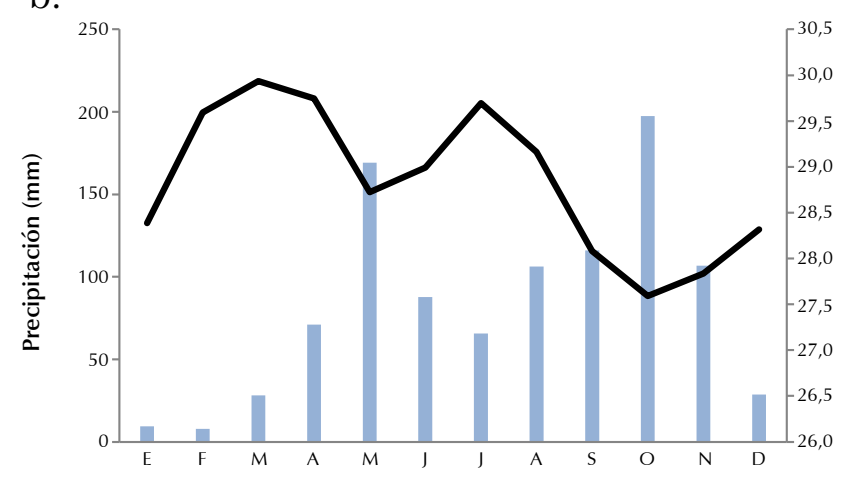

d.

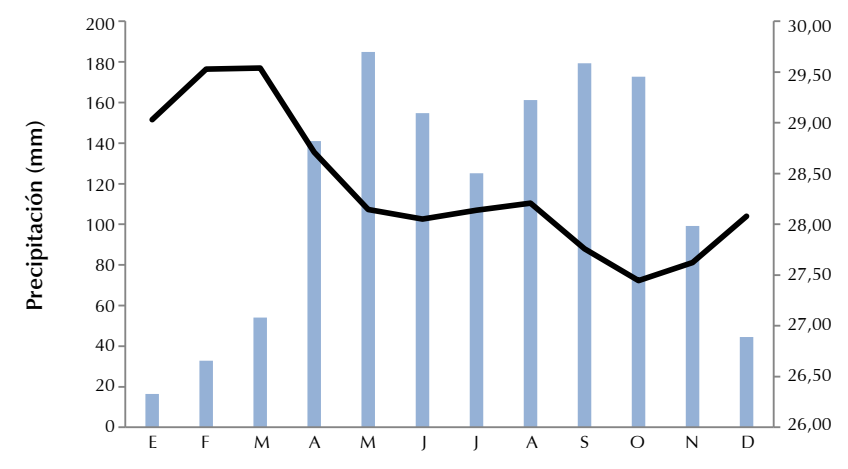

Figura 1. a. Fluctuación interanual de la precipitación estación Aeropuerto Alfonso López, Valledupar; b. Promedio de las lluvias mensuales para el periodo 1970-2013; c. Fluctuación interanual de la precipitación estación Aguas Claras, Aguachica; d. Promedio de las Iluvias mensuales para el periodo 1974-2011.

Nota: precipitación (barras azules) y temperatura (líneas negras). Las líneas horizontales representan el promedio histórico.

límite de 40 rodajas basales utilizados en otros estudios dendrocronológicos (y por la disponibilidad de muestras en las zonas de estudio). Las rodajas fueron pulidas con lijas de diferente granulometría $(36,50,80$ y 120$)$ para posibilitar la correcta visualización y la marcación de los anillos de crecimiento. Se comprobó que en ambas hubo dificultades para identificar sus anillos de crecimiento, situación que ocurre en algunas especies tropicales que reflejan condiciones climáticas locales y eventos ecológicos particulares. Por ello, para determinar un fechado inicial, se identificaron secciones donde se encontraron anillos estrechos en cada una de las rodajas, a los que vinculó con años secos, y mayores amplitudes en los anillos de crecimiento se relacionaron con años lluviosos.
Construcción de las cronologías. Para la medición de los anillos se utilizó una lupa estereoscópica AmScope SW-3T24Z acoplada a una mesa de medición Velmex TA4021H1-S6, con una precisión de $0.001 \mathrm{~mm}$. Para verificar los errores en la datación de los anillos de crecimiento se utilizó el programa COFECHA con los estadísticos intercorrelación de la serie (valor crítico 0.32) y sensibilidad media (Grissino-Mayer, 2001; Holmes, 1983). La estandarización de los anillos de crecimiento se realizó empleando el programa ARSTAN (Cook, 1985) con una curva exponencial negativa que transforma las mediciones de los anillos en índices de crecimiento. Este programa reduce la variación interanual causada por la tendencia biológica de formar anillos más angostos en la senectud y los 
disturbios endógenos, y maximiza el porcentaje de varianza común en el ancho de los anillos entre las series. También se evaluó: el intervalo común óptimo, que se refiere al mayor período de tiempo con el máximo número de series de índices de crecimiento; la correlación media entre radios, entre árboles, dentro de árboles y de cada radio con la cronología media; la relación señal-ruido, entendida como la robustez de la señal común observada entre los árboles que componen la cronología; y la señal expresada de la población (EPS), que es la concordancia con la cronología poblacional, encargada de cuantificar el grado de representación de la cronología obtenida (Briceño-J. et al., 2016; Speer, 2010).

Relación entre el índice de crecimiento y las variables ambientales. Se calculó el coeficiente de correlación de Pearson entre las variables climáticas con fluctuación mensual y los índices de crecimiento de los anillos. La relación estadística entre el ancho del anillo y las variables climáticas se examinó para el periodo común entre la cronología de los anillos de crecimiento y los datos instrumentales de precipitación y temperatura. Como el crecimiento en un año dado puede estar influenciado por las condiciones climáticas del año previo, el periodo de comparación analizado comprendió 24 meses (enero-diciembre) (Briceño-J., 2017). Esto se realizó con el fin de tener un registro climático más amplio y poder realizar así las comparaciones ambientales con los índices de crecimiento de las especies.

Para el SOI se calculó la correlación entre el crecimiento anual y los valores estimados por la National Oceanic and Atmospheric Administration (NOAA) en un periodo común desde enero a diciembre usando el programa RWizard (Guisande et al., 2014). Se consideraron niveles de significancia $\left(*, p<0.05 ;{ }^{* *}, p<0.01 y^{* * *}, p<0.001\right)$ (Briceño-J., 2017).

Modelos de crecimiento. Para obtener la función matemática que representó el crecimiento de las especies, se utilizó el programa RWizard (versión 4.3) (Guisande et al., 2014) con los modelos de regresión para variables dependientes cuantitativas mediante funciones no lineales (Clench, Exponencial negativa, Logístico y Gompertz). Al escoger el mejor modelo se tuvo en cuenta la normalidad de los residuos, el mayor valor del coeficiente de determinación, el menor error estándar residual y el menor número de iteraciones.

\section{RESULTADOS}

\section{Cronologías}

Aspidosperma polyneuron. De las 45 rodajas, 38 fueron seleccionadas y 7 presentaron dificultades en la datación para encontrar el límite del anillo de crecimiento, pues los anillos se agrupaban impidiendo la medición y su seguimiento alrededor de la rodaja no fue posible. Se midieron, a su vez, 2 radios por cada rodaja. Como resultado, la datación con el programa COFECHA permitió construir una cronología entre 1854 y 2014 (161 años) con una inter-correlación de la serie de 0.46 entre series y una sensibilidad media de 0.36. El mayor número de series fue 50 a partir de 1955, ya que el $65 \%$ de las muestras permitió esta amplitud de la cronología; el valor de EPS se estabilizó en 0.76 a partir de 1894 (Figura 2a).

Anacardium excelsum. Se seleccionaron 27 de las 45 rodajas y se descartaron 18 que mostraron manchas en las secciones impidiendo la correcta visualización y el marcaje de los anillos; además revelaron algunos radios con anillos comprimidos y radios con leño de reacción. Estas 18 rodajas presentaron problemas en la datación, con inter-correlación entre la serie por debajo de 0.32 . La cronología cubrió un periodo de 128 años, de 1887 a 2014. La inter-correlación entre series fue de 0.45 con una sensibilidad media de 0.41 .

El mayor número de series fue de 27 a partir de 1992 (100\% de las rodajas estudiadas) y el valor de EPS se estabilizó en 0.72 a partir de 1995 (Figura 2b). 


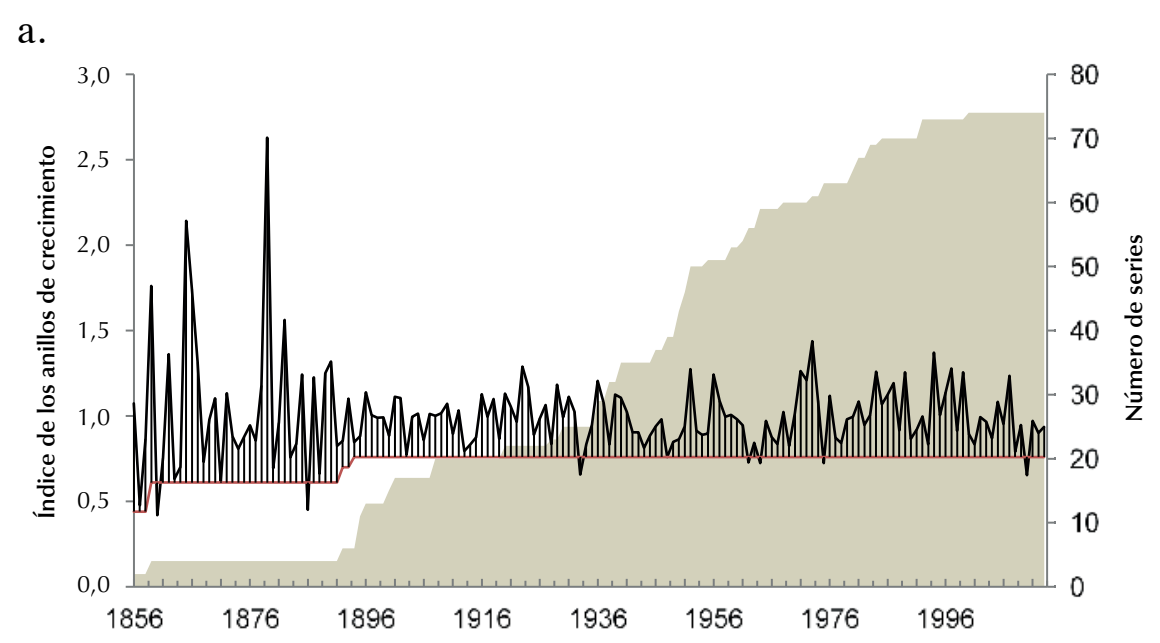

b.

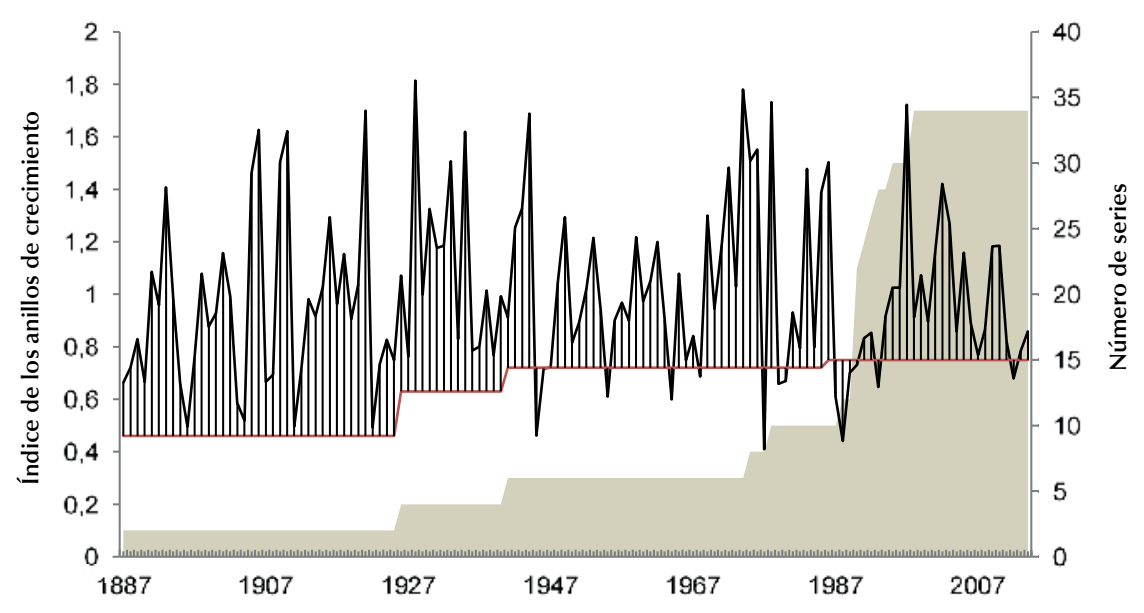

Figura 2. a. Cronología de Aspidosperma polyneuron (periodo 1856-2013) en Río de Janerio, Valledupar; b. Cronología de Anacardium excelsum (periodo 1887-2014) en la Bocatoma, Aguachica.

Nota: el área gris representa el número de series de la cronología y la línea roja el valor del EPS.

\section{Marcha anual de las variables climáticas y la formación de los anillos de crecimiento}

Aspidosperma polyneuron. El crecimiento mostró valores de correlación positiva (estadísticamente significativos) con la precipitación de enero $(r=0.55$, $p<0.001)$, de abril $(r=0.34, p<0.05)$ y de julio $(r=0.33$, $\mathrm{p}<0.05)$ del año anterior a la formación del anillo de crecimiento. Por el contrario, las correlaciones fueron negativas con noviembre $(r=-0.39, p<0.01)$ y diciembre $(r=-0.29, p<0.05)$ del año de formación del anillo de crecimiento (Figura 3a). La temperatura de enero tuvo una correlación negativa con significancia estadística $(r=-0.39, \mathrm{p}<0.01)$ y el SOI mensual no mostró valores significativos (Figura 3a).

Anacardium excelsum. El crecimiento mostró valores significativos positivos con la precipitación de octubre $(r=0.32, p<0.05)$ del año previo a la formación del anillo de crecimiento y con la de marzo $(r=0.54$, $\mathrm{p}<0.01)$ del año de formación del anillo de crecimiento; expresión similar se obtuvo con la temperatura del mes de octubre $(r=0.32, p<0.05)$. Con relación al SOI, los valores de los anillos de crecimiento no mostraron relación significativa (Figura 3b). 
a.

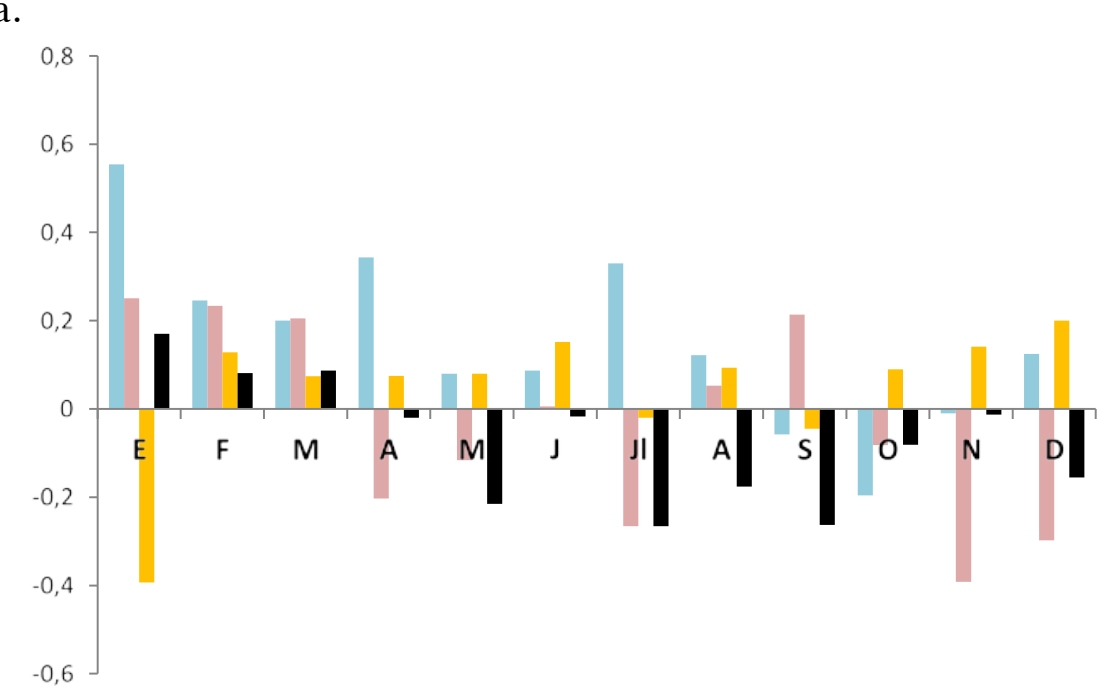

b.

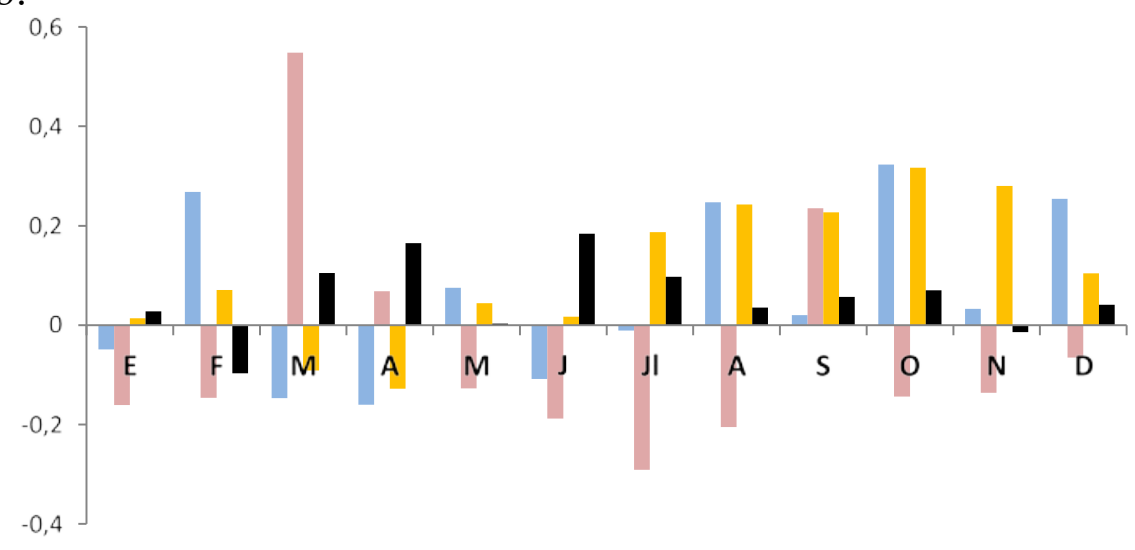

Figura 3. a. Correlación de los índices de crecimiento de Aspidosperma polyneuron en Río de Janeiro, Valledupar; b. Correlación de los índices de crecimiento de Anacardium excelsum en la Bocatoma, Aguachica.

Nota: las barras azules indican la precipitación del año anterior, las rosadas la precipitación del año actual, las amarillas la temperatura y las negras el SOI.

\section{Modelos de crecimiento}

Aspidosperma polyneuron. El modelo que mejor representó el crecimiento diamétrico acumulado de A. polyneuron fue la función de Gompertz (Figura 4a). Los coeficientes de la ecuación fueron significativos para el modelo $(p<0.05)$, el coeficiente de determinación fue 0.99 y el error estándar de la ecuación fue 6.87, con ocho (8) iteraciones; los datos fueron normales de acuerdo con la prueba de Lilliefors (Kolmogorov-Smirnov). La ecuación que representó el crecimiento de la especie fue:

$$
C r=196.3 * e^{-2.8^{*} e^{-0.02 * t}}
$$

Donde:

Cr: crecimiento diamétrico acumulado en $\mathrm{cm}$ y t: tiempo transcurrido en años.

Anacardium excelsum. El modelo que mejor representó el crecimiento diamétrico acumulado de 
A. excelsum fue la función logística (Figura 4b). Los coeficientes de la ecuación fueron significativos para el modelo $(p<0.05)$, el coeficiente de determinación fue 0.98 y el error estándar de la ecuación fue 20.14, con nueve (9) iteraciones; los residuos del modelo no fueron normales de acuerdo a la prueba de Lilliefors (Kolmogorov-Smirnov) ( $p=0.003)$. La ecuación que representó el crecimiento de la especie fue:

$$
C r=\frac{605.3}{1+7.9 * e^{\left(-0.04^{*} t\right)}}
$$

\section{Donde:}

Cr: crecimiento diamétrico acumulado en cm y t: tiempo transcurrido en años.

\section{DISCUSIÓN}

Los resultados confirman el potencial de las especies tropicales, especialmente de $A$. polyneuron y A. excelsum, para el desarrollo de estudios dendrocronológicos (en extensión, validación y estadísticos de la cronología). También, la utilidad de los anillos de crecimiento para documentar la variación climática en el bosque seco, como lo reportado en Cordia alliodora (Ruiz \& Pav.) Oken, Pseudobombax septenatum (Jacq.) Dugand, Annona spraguei Saff. en Costa Rica (Devall et al., 1995), Prosopis pallida (Willd.) Kunth (López-B. et al., 2005) y Bursera graveolens (Kunth) Triana \&

a.

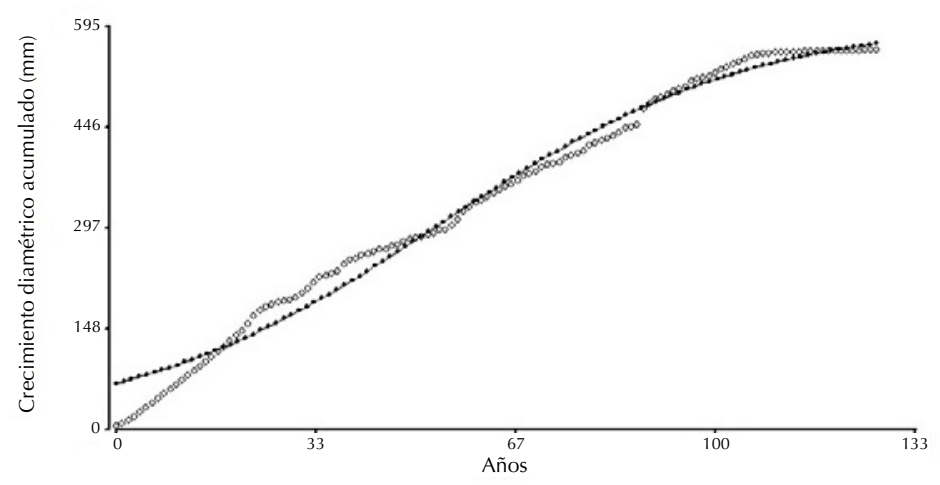

b.

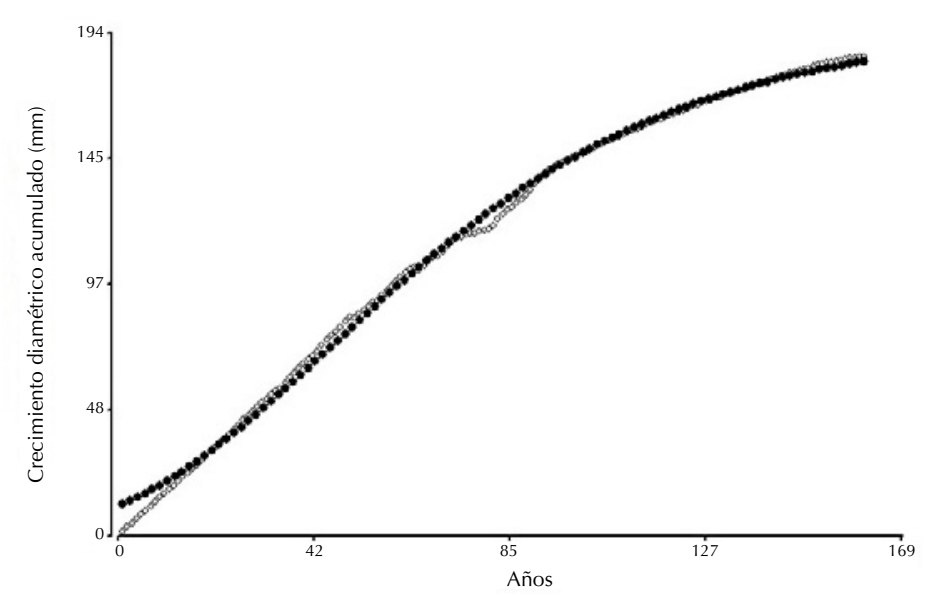

Figura 4. a. Modelo de crecimiento diamétrico acumulado de Aspidosperma polyneuron en Río de Janeiro, representado por la función Gompertz; b. Modelo de crecimiento diamétrico acumulado de Anacardium excelsum en la Bocatoma, representado por la función logística.

Nota: los círculos grises corresponden a los datos y los negros al modelo. 
Planch (Rodríguez et al., 2005) en Perú, así como Capparis odoratissima Jacq en los bosques secos de la Guajira colombiana (Ramírez y Del Valle, 2011).

Para A. polyneuron y A. excelsum los valores de la señal común expresada de la población (EPS) fueron 0.76 y 0.75 respectivamente, resultados similares al encontrado en Cordia alliodora (Ruiz \& Pav.) Oken (0.7) en un bosque seco tropical de Colombia (Briceño-J. et al., 2016), aunque ligeramente inferiores al valor recomendado $\geq 0.85$ (Wigley et al., 1984). Otros estudios han mostrado valores más altos en el EPS, relacionándolo con la intensidad de muestreo 30-40 muestras basales. Sin embargo, el EPS es sensible a las particularidades de cada sitio, por ejemplo, valores por debajo de 0.85 pueden asociarse a condiciones como pendientes y presión antrópica (López y Villalba, 2011). De esta manera el resultado de menor valor de referencia puede asociarse a la ocurrencia de incendios forestales, no solo por la prolongación de las sequías en los bosques estudiados, sino por los cambios en el uso del suelo para actividades agrícolas y prácticas culturales que han desencadenado procesos erosivos hasta la desertificación (Díaz et al., 2003).

\section{Los anillos de crecimiento y las variables ambientales}

\section{Precipitación y temperatura}

Los anillos de crecimiento de A. polyneuron se relacionaron con la precipitación evidenciado un crecimiento positivo en la época de inicio de las lluvias y durante el periodo seco cuando se presentaron lluvias espontáneas, situación similar a la encontrada en Tachigali myrmecophila (Ducke) Ducke en un bosque de Manaus $\left(3^{\circ} \mathrm{S}, 60^{\circ} \mathrm{W}\right)$ donde la correlación fue positiva entre el crecimiento y la precipitación (Ballantyne et al., 2011). La influencia positiva de las Iluvias durante el periodo seco encontrado en A. polyneuron se documentó en especies africanas de Acacia (Gourlay, 1995).

Los valores altos en la temperatura media y la escasa precipitación (por ejemplo, en enero) limitan el crecimiento de A. polyneuron: se encontraron valores negativos en las correlaciones estadísticamente significativas, por lo que en años más cálidos con incrementos de la evapotranspiración y del déficit hídrico es de esperar mayores limitaciones. Patrones similares en el crecimiento de los anillos de Amburana cearensis (Allemăo) A.C.Sm. del bosque seco en Bolivia $\left(16^{\circ} 9^{\prime} \mathrm{S}, 60^{\circ} 47^{\prime} \mathrm{W}\right)$ fueron mencionados por Paredes-Villanueva et al. (2015).

En el caso de $A$. excelsum el crecimiento se encuentra positivamente influenciado por los meses más lluviosos del año (octubre, un mes típicamente Iluvioso) y por marzo, al inicio de la temporada de Iluvias. Estos resultados se pueden asociar a la marcada dependencia que muestra la especie frente a la disponibilidad de agua, así como su adaptación a los regímenes de la formación del bosque tropical seco (Rangel-Ch., 2012). La correlación positiva significativa del crecimiento con la temperatura fue la de octubre, mes con el valor más bajo de temperatura en toda la serie mensual multianual $\left(27.4^{\circ} \mathrm{C}\right)$.

\section{Índice SOI}

Los anillos de crecimiento de A. polyneuron y $A$. excelsum no mostraron correlaciones significativas con el SOI, resultado similar al encontrado en Bursera graveolens en un bosque seco del Ecuador (reserva Catamayo, 422's, 7990’W) (Pucha-Cofrep et al., 2015). Así pues, este resultado refuerza las consideraciones sobre el alcance geográfico de los fenómenos climáticos como El Niño y La Niña y sus efectos positivos o negativos sobre el crecimiento de las especies (Briceño-J. et al., 2016).

\section{Modelos de crecimiento}

Los resultados de los modelos estadísticos del crecimiento diamétrico de las especies estuvieron expresados por la curva logística para $A$. excelsum en Aguachica y por la función de Gompertz para $A$. polyneuron en Valledupar. Modelos sigmoidales similares mostró Machaerium scleroxylon en un bosque seco tropical en Bolivia (Paredes-Villanueva et al., 2013). Según los resultados, las especies 
estudiadas alcanzarán su diámetro máximo (asintótico) de la siguiente manera: A. polyneuron en 169 años $(194 \mathrm{~cm})$ en Valledupar y $A$. excelsum en 133 años $(596 \mathrm{~cm}$ ) en Aguachica. Otras especies en bosques tropicales han reportado un comportamiento similar: Cedrelinga catenaeformis (Ducke) Ducke, Cedrela odorata L, Amburana cearensis (Allemăo) A.C.Sm y Peltogyne heterophylla M.F. Silva (Brienen y Zuidema, 2006).

Los análisis efectuados indican que las acciones extractivas de la madera de estas especies en los bosques secos tienen como consecuencia que la recuperación o la restauración en bosques dominados por estas especies tardarán entre 133 y 169 años para que alcancen su mayor diámetro (asintótico).

Teniendo en cuenta este tiempo, se recomienda a las entidades gubernamentales poner en marcha programas de conservación de áreas con parches originales de esta vegetación en la región del Caribe colombiano (Briceño-J, 2017). Así como detener los procesos de avance de la deforestación de los bosques secos y la implementación de estrategias de deforestación evitada (Rangel-Ch., 2012).

\section{CONCLUSIONES}

El crecimiento de A. polyneuron está influenciado positivamente por la precipitación en los meses previos a la formación del anillo de crecimiento $y$ en los meses en los cuales se presentan precipitaciones espontáneas en la época seca; para A. excelsum esto ocurre con el mes de mayor precipitación.

La temperatura no mostró correlaciones significativas (estadísticamente) con el crecimiento de $A$. polyneuron y A. excelsum.

El Índice de Oscilación del Sur (SOI) no mostró correlaciones significativas (estadísticamente) con el crecimiento de las especies estudiadas.

Los anillos de crecimiento de Aspidosperma polyneuron y Anacardium excelsum, reflejan la influencia de las variaciones locales de precipitación media mensual y de temperatura media mensual.
El crecimiento diamétrico máximo que alcanzarán Aspidosperma polyneuron y Anacardium excelsum en los bosques secos tropicales tardará más de 100 años.

\section{AGRADECIMIENTOS}

Los autores agradecen a Minciencias (Colombia) y a la Universidad Nacional de Colombia.

\section{CONFLICTO DE INTERESES}

Los autores declaran no tener conflicto de intereses.

\section{CONTRIBUCIÓN POR AUTOR}

Los dos autores contribuyeron planificando la investigación, ejecutándola y en la preparación del manuscrito.

\section{REFERENCIAS}

Arias, D. (2005). Morfometría del árbol en plantaciones forestales tropicales. Kurú Revista Forestal Costa Rica, 2(5),1-13.

Ballantyne, A., Baker, P., Chambers, J., Villalba., R. y Argollo., J. (2011). Regional differences in South American Monsoon precipitation inferred from the growth and isotopic composition of tropical trees. Earth Interactions, 15(5), 1-35.

https://doi.org/10.1175/2010El277.1

Bendix, J., Trache, K., Palacios, E., Rollenbeck, R., Goettlicher, D., Nauss, T. y Bendix, A. (2011). El Niño meets La Niña - anomalous rainfall patterns in the "traditional" El Niño region of southern Ecuador. ERDKUNDE, 65(2), 151-167. https://doi.org/10.3112/erdkunde.2011.02.04

Berliner, M. (2003). Uncertainty and climate change. Statistical Science, 18(4), 430-435. https://doi.org/10.1214/ss/1081443227 
Briceño-J, A. M. (2017). Dinámica del crecimiento y relación con el clima de especies arbóreas de los bosques de la región Caribe, Colombia [Tesis doctoral, Universidad Nacional de Colombia]. Repositorio institucional Universidad Nacional de Colombia.

https://repositorio.unal.edu.co/handle/unal/59369

Briceño-J, A. M., Rangel-Ch, J. O y Bogino, S. (2016). Estudio de los anillos de crecimiento de Cordia alliodora (Ruiz \& Pav.) Oken en Colombia. Colombia Forestal, 19(2), 219-232.

https://doi.org/10.14483/udistrital.jour.colomb. for.2016.2.a07

Brienen, R. J. W. y Zuidema, P. A. (2006). The use of tree rings in tropical forest management: Projecting timber yields of four Bolivian tree species. Forest ECOlogy and Management, 226 (1-3), 256-267. https://doi.org/10.1016/j.foreco.2006.01.038

Cook, E. R. (1985). A time series analysis approach to treering standardization [Tesis doctoral, Universidad de Arizona]. Repositorio institucional Universidad de Arizona.

https://trr.arizona.edu/content/time-series-analysis-approach-tree-ring-standardization

Del Valle, A. J. I. (1997). Crecimiento de cuatro especies de los humedales forestales del litoral pacífico colombiano. Revista de la Academia Colombiana de Ciencias Exactas, Físicas y Naturales, 21(81), 445-466.

Devall, M. S., Parresol, B. R. y Wright, S. J. (1995). Dendroecologycal analysis of Cordia alliodora, Pseudobombax septenatum and Annona spraguei in central Panama. IAWA Journal, 16(4), 411-424.

https://doi.org/10.1163/22941932-90001430

Díaz, D. R., Lloret, F. y Pons, X. (2003). Influence of fire severity on plant regeneration through remote sensing imagery. Internationa Journal of Remote Sensing, 24(8), 1751-1763.

https://doi.org/10.1080/01431160210144732

Fedorov, A. V. y Philander, S. G. (2000). Is El Niño changing? Science, 288(5473), 1997-2002.

https://doi.org/10.1126/science.288.5473.1997

Gourlay, I. D. (1995). Growth ring characteristics of some African Acacia species. Journal of Tropical Ecology, 11(1), 121-140.

https://doi.org/10.1017/S0266467400008488
Grissino-Mayer, H. D. (2001). Evaluating crossdating accuracy: a manual and tutorial for the computer program COFECHA. Tree-ring Research, 57(2), 205-221.

Groenendijk, P., Sass-Klaassen, U., Bongers, F. y Zuidema, P. A. (2014). Potential of tree-ring analysis in a wet tropical forest: a case study on 22 commercial tree species in Central Africa. Forest Ecology and Management, 323(1), 65-78.

https://doi.org/10.1016/j.foreco.2014.03.037

Guisande, C., Heine, J., González-DaCosta, J. y García-Roselló, E. (2014). RWizard (Versión 4.3) [Software de computador]. University of Vigo.

Holmes, R. L. (1983). Computer-assisted quality control in tree-ring dating and measurement. Tree-Ring Bull, 43, 69-75.

Hughes, M. K. (2011). Dendroclimatology in High-Resolution Paleoclimatology. Dendroclimatology Progress and Prospects, 364, 17-34.

López, L. y Villalba, R. (2011). Climate influences on the radial growth of Centrolobium microchaete, a valuable timber species from the tropical dry forests in Bolivia. Biotropica, 43(1), 41-49.

https://doi.org/10.1111/j.1744-7429.2010.00653.x

López-B, C., Sabaté, S., García, C. A. y Rodríguez, R. (2005). Wood anatomy, description of annual rings, and responses to ENSO events of Prosopis pallida H.B.K., a wide-spread woody plant of arid and semi-arid lands of Latin America. Journal of Arid Environments, 61(4), 541-554.

https://doi.org/10.1016/j.jaridenv.2004.10.008

Malhi, Y. y Wright, J. (2004). Spatial patterns and recent trends in the climate of tropical rainforest region. Philosophical Transactions Biological Sciences, 359(1443), 311-329.

https://doi.org/10.1098/rstb.2003.1433

Minorta-C, V., Rangel-Ch, J. O., Castro-L, F. y Aymard, G. (2019). La vegetación de la serranía de Manacacías (Meta) Orinoquía colombiana. En J. O. Rangel-Ch (Ed.), Colombia Diversidad Biótica XVII: la región de la Serranía de Manacacías (Meta) Orinoquía colombiana (pp. 155-234). Universidad Nacional de Colombia-Instituto de Ciencias Naturales/Parques Nacionales Naturales de Colombia. 
Muñoz, F. H., Coria, V. M. A., García, J. J. S. y Balam, M. C. (2009). Evaluación de una plantación de tres especies tropicales de rápido crecimiento en Nuevo Urecho, Michoacán. Revista Ciencia Forestal en México, 34(106), 61-87.

Oliveira, J. M., Santarosa, E., Pillar, V. D. P. y Roig, F. A. (2009). Seasonal cambium activity in the subtropical rain forest tree Araucaria angustifolia. Trees, 23, 107-115. https://doi.org/10.1007/s00468-008-0259-y

Paredes-Villanueva, K., Sánchez-Salguero, R., Manzanedo, R. D., Quevedo-Sopepi, R., Palacios, G. y Navarro-Cerrillo, R.M. (2013). Growth rate and climatic response of Machaerium scleroxylon in a dry tropical forest in southeastern Santa Cruz, Bolivia. Tree-Ring Research, 69(2), 63-79.

https://doi.org/10.3959/1536-1098-69.2.63

Paredes-Villanueva, K., López, L., Brookhouse, M. y Navarro, R. M. C. (2015). Rainfall and temperature variability in Bolivia derived from the tree-ring width of Amburana cearensis (Fr. Allem.) A.C. Smith. Dendrochrologia, 35, 80-86. https://doi.org/10.1016/j.dendro.2015.04.003

Pereyra, M. J. E., Inga, G. J. G., Morales, M. S. y Rodríguez, R. A. (2014). Potencialidad de Cedrela odorata (Meliaceae) para estudios dendrocronológicos en la selva central de Perú. Revista Biología Tropical, 62(2), 783-793.

https://doi.org/10.15517/rbt.v62i2.9835

Pucha-Cofrep, D., Peters, T. y Bräuning, A. (2015). Wet season precipitation during the past century reconstructed from tree-rings of a tropical dry forest in Southern Ecuador. Global and Planetary Change, 133, 65-78.

https://doi.org/10.1016/j.gloplacha.2015.08.003

Ramírez, J. A. y Del Valle, J. I. (2011). Paleoclima de la Guajira, Colombia: según los anillos de crecimiento de Capparis odoratissima (Capparidaceae). Revista Biología Tropical, 59(3), 1389-1405.

Rangel-Ch, J. O. (2012). La vegetación de la región Caribe de Colombia: composición florística y aspectos de la estructura. En J. O. Rangel-Ch. (Ed.), Colombia Diversidad Biótica XII: la región Caribe de Colombia (pp. 365-476). Universidad Nacional de Colombia-Instituto de Ciencias Naturales.

Rangel-Ch., J. O. y Carvajal-Cogollo, J. E. (2009). Clima de la Serranía del Perijá. En J. O. Rangel-Ch. (Ed.), Colombia Diversidad Biótica VIII: media y baja montaña de la Serranía de Perijá (pp. 3-35). Universidad Nacional de Colombia-Instituto de Ciencias Naturales.

Rangel-Ch., J. O. y Carvajal-Cogollo, J. E. (2012a). Suelos de la región Caribe de Colombia. En J. O. Rangel-Ch. (Ed.), Colombia Diversidad Biótica XII: la región Caribe de Colombia (pp. 879-921). Universidad Nacional de Colombia-Instituto de Ciencias Naturales.

Rangel-Ch., J. O. y Carvajal-Cogollo, J. E. (2012b). Clima de la región Caribe colombiana. En J. O. Rangel-Ch. (Ed.), Colombia Diversidad Biótica XII: la región Caribe de Colombia (pp. 6-129). Universidad Nacional de Colombia-Instituto de Ciencias Naturales.

Rodríguez, N. (2015). Implementación de una acción para la conservación y uso sostenible de Aspidosperma polyneuron (Mutis ex L.f.). Wess. Boer en la jurisdicción CAR. Corporación Autónoma Regional de Cundinamarca (CAR).

Rodríguez, R., Mabres, A., Luckman, B., Evans, M., Masiokas, M. y Ektvedt, T. M. (2005). "El Niño" events recorded in dry-forest species of the lowlands of northwest Peru. Dendrochronologia, 22(3), 181-186.

https://doi.org/10.1016/j.dendro.2005.05.002

Stahle, D. W., Mushove, P. T., Cleaveland, M. K., Roig, F. y Hayness, G. A. (1999). Management implications of annual growth ring in Pterocarpus angolensis from Zimbabwe. Forest Ecology and Management, 124(2-3), 217-229.

https://doi.org/10.1016/S0378-1127(99)00075-4

Speer, J.H. (2010). Fundamentals of Tree-Ring Research. The University of Arizona Press. 324p.

Suatunce, P. C., Díaz, G. C. y Cruzatty, L. G. (2009). Crecimiento de especies arbóreas tropicales en la colección de la Universidad Técnica Estatal de Quevedo. Ciencia y Tecnología, 2(2), 21-27. 
Tomazello-Filho, M., Botosso, P. C. y Lisi, C. (2000). Potencialidade da familia Meliaceae para estudos dendrocronológica em regioes tropicais e subtropicais. En F. A. Roig (Ed.), Dendrocronologia en América Latina (pp. 381-389). Editorial Universidad Nacional de Cuyo.

Tomazello-Filho, M., Roig, F. A. y Zevallos, P. A. (2009). Dendrocronología y dendroecología tropical: marco histórico y experiencias exitosas en los países de América Latina. Ecología en Bolivia, 44(2), 73-82.

Wigley, T. M. L., Briffa, K. R. y Jones, P. D. (1984). On the average value of correlated time series, with applications in dendroclimatology and hydrometeorology. Journal of Applied Meteorology and Climatology, 23(2), 201-213.

https://doi.org/10.1175/1520-0450(1984)023< 0201:OTAVOC>2.0.CO;2 